\title{
Novel Method for Assessing Moisture Damage in Asphalt Mixtures
}

\author{
Mohammad Nour Fakhreddine \\ mff17@mail.aub.edu \\ American University of Beirut, Beirut, Lebanon \\ Ghassan Chehab \\ gc06@aub.edu.lb \\ American University of Beirut, Beirut, Lebanon \\ Zaher Al Basiouni Al Masri \\ zsa14@mail.aub.edu \\ American University of Beirut, Beirut, Lebanon \\ Mohamad Abiad \\ ma192@aub.edu.lb \\ American University of Beirut, Beirut, Lebanon
}

\begin{abstract}
Moisture damage is a major cause of early pavement deterioration and often accelerates other distresses such as rutting, fatigue cracking, and raveling. Water infiltrates into the pavement structure through the cracks and air voids and weakens the adhesive bond between the binder and the aggregates as well as the cohesive bond between the binder particles. Several tests have been adopted to test for moisture susceptibility, most commonly the Modified Lottman (AASHTO, T283) and the Hamburg Wheel Tracking Device (AASHTO, T32404), however, these tests correlate poorly with field results and do not properly replicate the conditions which a pavement structure experiences in the field. Also, the mechanism which determines the mode of failure due to moisture damage, being either adhesive or cohesive, remains largely not understood, and the research tackling this issue is very scarce. The objective of this study is to introduce a new testing procedure based on the pull-off approach and study the factors which influence the mode of failure of the samples, such as the asphalt film thickness and loading rate.
\end{abstract}

Keywords: Moisture damage; Adhesion and cohesion; Stripping; Asphalt-aggregate bond; Strain rate; Asphalt film thickness

\section{INTRODUCTION}

Pavements constitute a major part of the transportation infrastructure network in most countries, providing invaluable social and economic benefits. The level of maintenance of a pavement network can have a significant impact on the economy and safety of users; as such, it is necessary to understand the causes of pavement deterioration to extend their lifetime and improve their performance under field conditions. Moisture damage is a major problem that affects the durability of pavements and causes premature deterioration, which in turn significantly increases the annual vehicle operating costs for users (Caro et al., 2008). It also accelerates the occurrence of other major distresses, such as rutting and cracking (Kakar, 2015). Failure can occur due to loss of adhesion and/or cohesion. The loss of adhesion, also known as stripping, is when the interfacial bond between the binder and aggregate surface deteriorates, whereas the loss of cohesion happens within 
the binder itself due to the decrease in stiffness (Diab et al., 2014). Moisture damage causes the aggregates to break loose from the structure, which over time, leads to the formation of potholes and cracks; these distresses decrease the safety and rideability of the road in addition to incurring high maintenance bills for municipalities and the users of the road. As such, a reliable and simple procedure that measures the moisture susceptibility of pavements is necessary.

\section{LITERATURE REVIEW}

The AASHTO T283 is most commonly used to measure the moisture susceptibility of asphalt mixtures. The test consists of placing 3 samples in a water bath for 24 hours at 60 degrees $\mathrm{C}$. The samples are then conditioned at 25 degrees $\mathrm{C}$ for 2 hours before being tested in indirect tension at a rate of 2 inches per minute. The results are compared to those of 3 samples which were not subject to moisture conditioning (AASHTO). However, the test uses compacted asphalt cylindrical samples and thus does not isolate the stripping phenomenon at the level of the asphalt-aggregate interface (Al Basiouni Al Masri et al., 2019). The test also uses a fixed conditioning time and load rate, which may not simulate accurate conditions experienced by the pavement in the field. Other tests such as the Hamburg Wheel Tracking Device (AASHTO, T324), Environmental Conditioning System (ECS) (AASHTO, TP34), Asphalt Pavement Analyzer (APA), and Moisture Induced Sensitivity Test (MIST) also exist but are rarely used due to the lack of standardization and complexity of the procedures and sample preparation (Taib et al., 2019).

Researchers have proposed other methods to evaluate moisture sensitivity by using the Dynamic Shear Rheometer (DSR) and different variations of pull-off tests. Cho and Bahia (2010) proposed the wet to dry yield shear stress (W/D YSS) ratio as a parameter to quantify moisture damage by using the Dynamic Shear Rheometer (DSR). They concluded that while the W/D YSS ratio is sensitive enough to measure moisture effects, other factors limit the use of the DSR. Zhang et al. (2016) also used the DSR to control the film thickness of the samples by adding specially manufactured parts for it. The samples were then tested using a Universal Testing Machine (UTM) by also using custom fixtures to hold the samples, which makes the testing setup both very expensive and complicated to use. Rahim et al. (2019) also modified a $10 \mathrm{KN}$ Universal Testing Machine (UTM) and the authors reported that further improvement for the gap assembly is needed for repeatability and practicability reasons. One of the most common tests to evaluate moisture damage is the Bitumen Bond Strength (BBS) test, which uses the Pneumatic Adhesion Tensile Testing Instrument (PATTI). The BBS test consists of pulling a metal stub attached to an aggregate substrate by a film of binder and recording the maximum pulling force. The test was reported by (Moraes et al., 2011) to be successful in measuring the effects of moisture conditioning timing and asphalt modification on the bond strength of asphalt-aggregate systems. The BBS test was used to investigate several issues such as the effect of the bitumen stiffness on the adhesive strength, (Moraes et al., 2012) and the adhesive and cohesive properties of asphalt-aggregate systems using different combinations of aggregates, binders, and mineral fillers (Canestrari et al., 2010; Chaturabong et al., 2018). Despite the popularity of the BBS test, it does have some drawbacks such as using a high film thickness of $0.8 \mathrm{~mm}$, not all elements of the system 
being at the same temperature at the time of the sample preparation, and the limitation of the test in terms of output where only the strength can be obtained while other data such as strain cannot (Cala et al., 2019).

Although significant research has been done to evaluate the effect of moisture on the asphalt-aggregate bond using several variations of pull-off and direct tension tests and taking into consideration factors such as binder modification, soaking time, and aggregate mineralogy, little has been done to investigate the effect of test-related factors on the strength of the asphalt-aggregate bond. Such factors include the asphalt film thickness and the rate of loading.

\section{OBJECTIVES}

The objective of this study is to investigate the factors that affect the asphalt aggregate bond such as the asphalt film thickness and loading rate by running sensitivity analyses. The factors will be tested based on the direct tension test using a simple testing and sample preparation procedure. The results of this sensitivity study on the key affecting factors are used to come up with optimal testing and conditioning procedures that promote adhesive failure. The authors believe that the resulting test procedure will pave the way into really understanding the phenomenon of moisture damage in asphalt pavements through analyzing the load-displacement patterns and observing the modes of failure at the level of the asphalt-aggregate interfacial bond.

\section{MATERIALS}

For the purpose of the sensitivity study, two types of asphalt binder are used:

1) An unmodified PG64-16 binder

2) A SBS polymer modified PG76-28 binder.

Along with limestone aggregates. Furthermore, limestone filler (less than $75 \mu \mathrm{m}$ particle size) is mixed with both binders to produce asphalt mastics. As part of the ongoing research, the use of filler material from construction demolition waste (CDW) is being investigated. Finally, the effect of using recycled coarse aggregates, namely recycled concrete aggregates (RCA) and reclaimed asphalt pavement (RAP), on moisture susceptibility of asphalt-aggregate systems is also being studied but is not presented in this paper.

\section{SAMPLE PREPARATION AND TEST PROCEDURE}

To produce the asphalt-aggregate samples used in the tensile test, limestone cylinders are first produced by coring large limestone blocks obtained from a known quarry. Then, the cylinders are sliced into 5mm-thick aggregate discs, each of which is epoxied into an aluminum endplate. The exposed aggregate surface is cleaned using a clean cloth dipped in a small amount of acetone to remove any residues or oils that might affect the adhesion with the asphalt binder. Meanwhile, asphalt binder is heated until it liquefies, poured in a round silicon mold, and left to cool down at room temperature. In order to control the asphalt film thickness, the zero-gap is defined by fixing two aggregate discs into the tensile machine and lowering the actuator until a $1 \mathrm{~N}$ contact load is recorded.

The aggregates are then removed and placed on top of a hot plate to reach the binder application temperature which should be similar to the mixing temperature for an asphalt 
mix with the same binder. After the temperature of the aggregates stabilizes, they are quickly fixed in the tensile machine and the binder disc is directly attached to the hot aggregate surface. The actuator is then lowered until the specified film thickness is reached. Finally, the excess binder is trimmed, and the sample is left to cool down to room temperature before testing or removing from the machine. The temperature of the sample is monitored using a thermocouple inserted at the middle of a dummy specimen; it was found that 30 minutes are enough to bring the entire sample back to room temperature. It should be noted that the time between heating the aggregates and lowering the actuator should not be more than 1 minute to avoid excessive loss of temperature of the aggregate surface, which can cause inadequate adhesion with the binder. The sample preparation procedure is visually summarized in Figure 1.

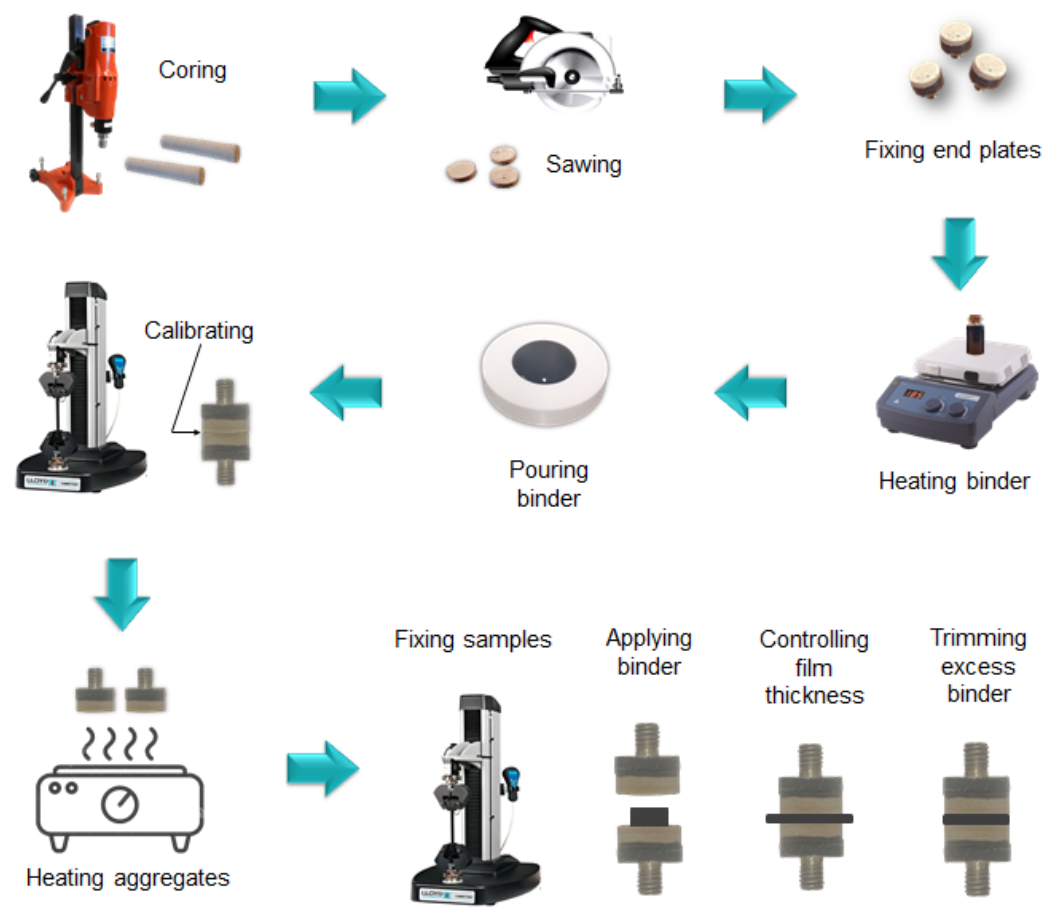

Figure 1: Sample Preparation Procedure

Three replicates of each asphalt-aggregate combination for each testing condition are fabricated using the procedure detailed above.

\subsection{Problems Faced with Sample Preparation and Testing}

After several trials with the proposed testing procedure, the following challenges were faced and tackled:

- For samples that were tested after moisture conditioning, it was observed that some gained strength. The issue was traced back to the resting time after the samples were fabricated. The asphalt-aggregate bond continued to gain strength even after cooling to room temperature and seemed to stabilize after 24 hours. As such, it is recommended that all samples be left to rest for at least 24 hours before dry testing 
or moisture conditioning.

- Due to the viscoelastic nature of asphalt, the binder film decreased in thickness under the weight of the top plate and aggregate over the resting period. The decreased film thickness affected the strength of the bond and thus all samples were modified after fabrication by adding fixtures to their sides so that no load is applied on the asphalt film.

- Some samples exhibited low strength after testing due to the low temperature at which the binder was attached to the aggregate. It is necessary to ensure that the aggregates are sufficiently hot to allow for proper adhesion of the binder to the surface of the aggregates.

- Upon observation of the aggregate surface after testing, it was noticed that some samples were failing in adhesion particularly from one edge of the disk. This was because the surfaces of the 2 aggregates were not completely parallel and thus the sample had a tapered film thickness. The surfaces of the aggregates must be parallel to ensure a uniform film thickness.

\section{RESULTS}

\subsection{Effect of Rate of Loading}

The behavior of asphalt, being a viscoelastic material, depends on the strain rate being applied to it. It is known that as the strain rate increases, the stiffness of the binder also increases, and vice versa; and given that a pavement in the field experiences different strain rates depending on the speed of passing trucks and heavy vehicles, then it makes sense to test for different strain rates when checking for the moisture susceptibility of an asphalt mix. To verify that the strain rate influences the strength of the asphalt-aggregate bond and its mode of failure, 3 replicates were tested for each of the chosen strain rates and the results are as shown in Figure 2.

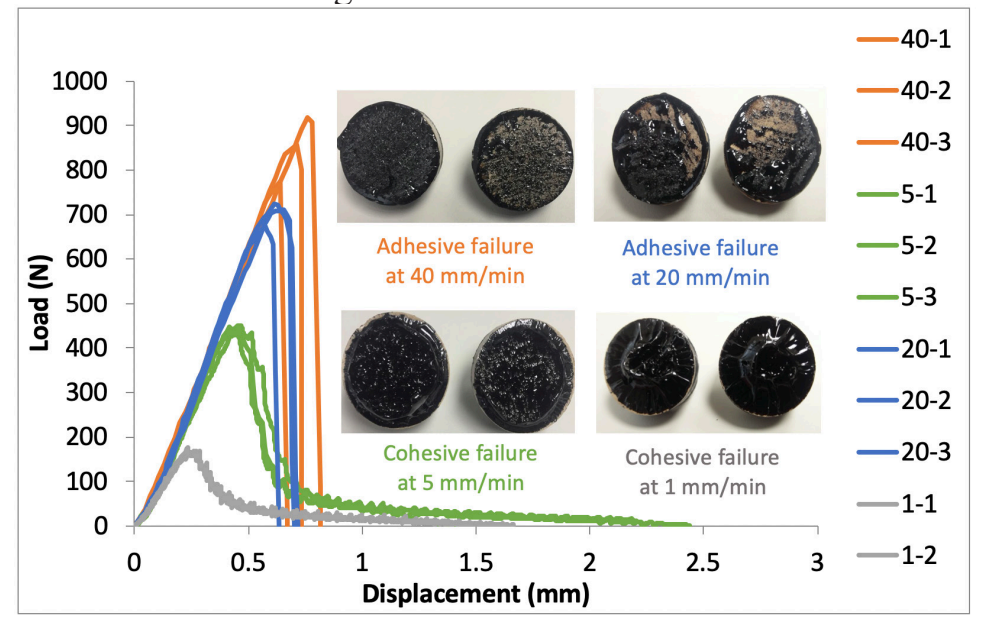

Figure 2: Load vs. Displacement and mode of failure for Different Strain Rates

As the strain rate increases, the strength of the asphalt-aggregate bond also increases. The mode of failure also tends to be adhesive for higher strain rates and cohesive for low strain rates. The test is thus very sensitive to the strain rate and it is a very important factor that can be manipulated to simulate field conditions and promote adhesive failure. 


\subsection{Effect of the Asphalt Film Thickness}

To check the sensitivity of the test for the asphalt film thickness, thicknesses of 0.1 $\mathrm{mm}, 1 \mathrm{~mm}$, and $1.5 \mathrm{~mm}$ were tested for samples using unmodified binder (Figure 3), polymer modified binder (Figure 4) and mastic (Figure 5) using the unmodified binder and limestone filler for a ratio of 1 to 1 by mass. The samples were tested at a strain rate of $20 \mathrm{~mm} / \mathrm{min}$.

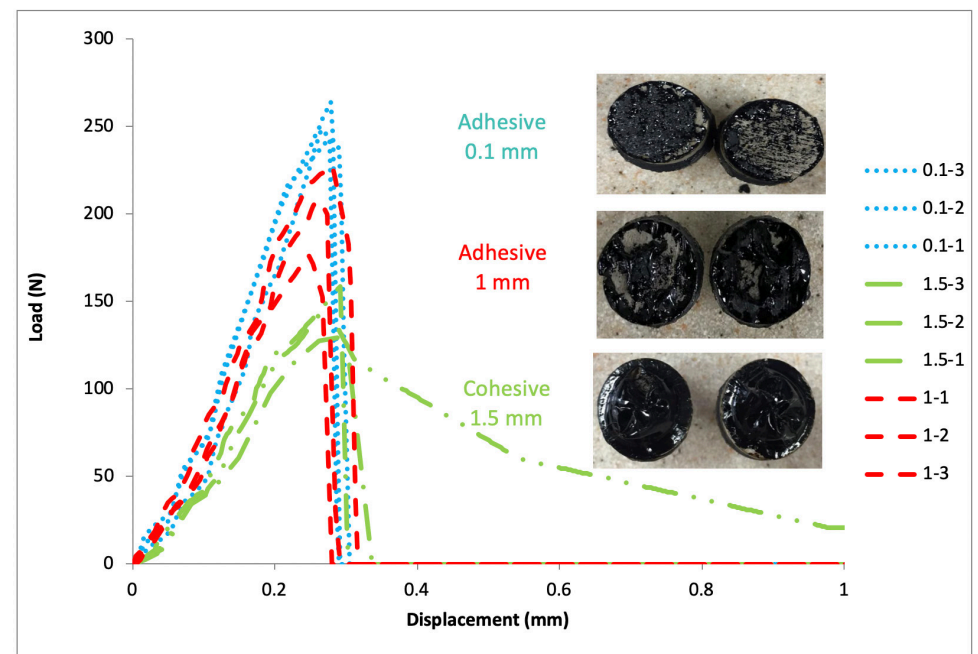

Figure 3: Load vs. Displacement and mode of failure for Different Film Thicknesses using Unmodified Binder

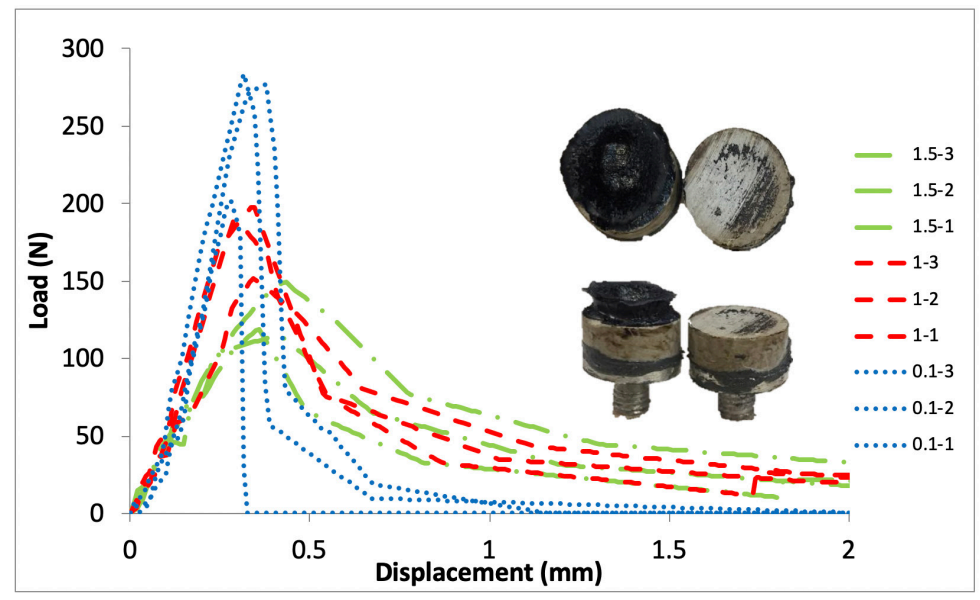

Figure 4: Load vs. Displacement and mode of failure for Different Film Thicknesses using Polymer Modified Binder 


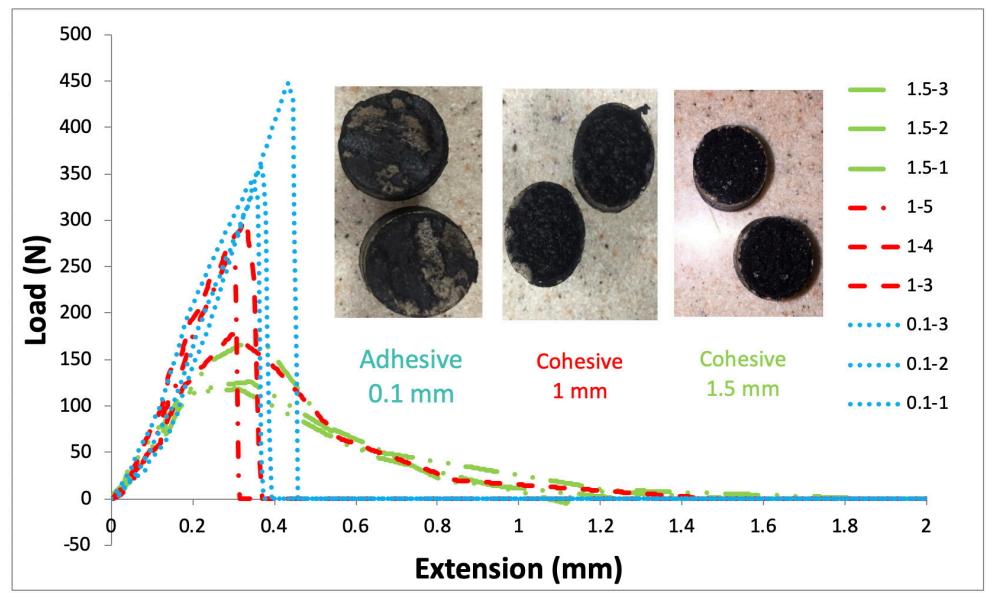

Figure 5: Load vs. Displacement and mode of failure for Different Film Thicknesses using Asphalt Mastic

The results show that as the asphalt film thickness decreases, the strength of the asphalt-aggregate bond increases and the mode of failure tends to be adhesive, while for higher film thicknesses, the strength decreases, and the mode of failure tends to be cohesive. In the case of the polymer-modified binder, the same mode of failure, which was completely adhesive, was observed for all film thicknesses, the authors suspect that the wetting temperature is the main reason behind this observation and will follow up on this issue in future work.

The results agree with (Huang \& Lv, 2016) who tested for different stress rates and film thicknesses but using the BBS test; Therefore, the authors were not able to collect load-displacement data, but only the strength of the samples at failure.

\section{CONCLUSION}

This study confirmed that the asphalt film thickness and loading rate are important factors that must be considered when measuring the moisture susceptibility of asphalt mixes due to their significant effect on the asphalt-aggregate bond. An increase in the loading rate increases strength and promotes adhesive failure, and an increase in asphalt film thickness decreases strength and promotes cohesive failure. The study also proposed a simple testing procedure where film thickness and strain rate can be easily adjusted, and additional data to the maximum strength can be obtained.

The authors believe that strain rate and asphalt film thickness should be included as parameters when testing for moisture susceptibility and will follow up this study with a more detailed one which includes testing of soaked samples.

\section{REFERENCES}

Al Basiouni Al Masri, Z., Alarab, A., Chehab, G. \& Tehrani-Bagha, A. (2019). Assessing moisture damage of Asphalt-Aggregate Systems using principles of Thermodynamics: Effects of recycled materials and binder aging. Journal of Materials in Civil Engineering, 31(9), 4019190. 
Cala, A., Caro, S., Lleras, M. \& Rojas-Agramonte, Y. (2019). Impact of the chemical composition of aggregates on the adhesion quality and durability of asphalt-aggregate systems. Construction and Building Materials, 216, 661-672.

Canestrari, F., Cardone, F., Graziani, A., Santagata, F. A. \& Bahia, H. U. (2010). Adhesive and cohesive properties of Asphalt-Aggregate Systems subjected to moisture damage. Road Materials and Pavement Design, 11(sup1), 11-32.

Caro, S., Masad, E., Bhasin, A. \& Little, D. N. (2008). Moisture susceptibility of asphalt mixtures, Part 1: mechanisms. International Journal of Pavement Engineering, 9(2), 81-98.

Chaturabong, P. \& Bahia, H. U. (2018). Effect of moisture on the cohesion of asphalt mastics and bonding with surface of aggregates. Road Materials and Pavement Design, 19(3), 741-753.

Cho, D.-W. \& Bahia, H. U. (2010). New parameter to evaluate moisture damage of AsphaltAggregate bond in using dynamic shear rheometer. Journal of Materials in Civil Engineering, 22(3), 267-276.

Diab, A., You, Z., Hossain, Z. \& Zaman, M. (2014). Moisture susceptibility evaluation of nanosize hydrated lime-modified Asphalt-Aggregate Systems based on surface Free Energy Concept. Transportation Research Record, 2446(1), 52-59.

Huang, W. \& Lv, Q. (2016). Investigation of critical factors determining the accuracy of binder bond strength test to evaluate adhesion properties of asphalt binders. Journal of Testing and Evaluation, 45(4), 1270-1279.

Moraes, R., Velasquez, R. \& Bahia, H. (2012). The Effect of Bitumen Stiffness on the Adhesive Strength Measured by the Bitumen Bond Strength Test.

Moraes, R., Velasquez, R. \& Bahia, H. U. (2011). Measuring the effect of moisture on asphaltaggregate bond with the bitumen bond strength test. Transportation Research Record, 2209(1), 70-81.

Rahim, A., Thom, N. \& Airey, G. (2019). Development of compression pull-off test (CPOT) to assess bond strength of bitumen. Construction and Building Materials, 207, 412-421.

Taib, A., Jakarni, F. M., Rosli, M. F., Yusoff, N. I. M. \& Aziz, M. A. (2019). Comparative study of moisture damage performance test. IOP Conference Series: Materials Science and Engineering, 512(1), 12008. IOP Publishing.

Zhang, J., Apeagyei, A. K., Grenfell, J. \& Airey, G. D. (2016). Experimental study of moisture sensitivity of aggregate-bitumen bonding strength using a new pull-off test. 8th RILEM International Symposium on Testing and Characterization of Sustainable and Innovative Bituminous Materials, 719-733. Springer. 